\title{
EFEITOS DA MISTURA PRONTA DE AMETRYNE MAIS SIMAZINE COM GLYPHOSATE E PARAQUAT NO CONTROLE DE PLANTAS DANINHAS NA CULTURA DO CAFEEIRO
}

\author{
Joaquim A. Carvalho', Maira A. N. Almeida ${ }^{2}$, Vera L. M. Santos ${ }^{2}$, Césio H. Brito ${ }^{3}$, \\ Carlos M. Santos ${ }^{2}$ \\ ${ }^{1}$ Universidade Federal de Uberlândia - UFU, Instituto de Ciências Agrárias - ICIAG, Uberlândia, MG. \\ ${ }^{2}$ Discente do Curso de Agronomia, UFU / ICIAG, Uberlândia, MG. \\ ${ }^{3}$ SYNGENTA. CP. 585, 38406.270, Uberlândia-MG
}

\begin{abstract}
RESUMO
Este trabalho foi realizado de 06/01 a 08/03/98 em Iraí de Minas, com objetivo de avaliar a atividade biológica da mistura pronta de ametryne mais simazine com glyphosate e paraquat no controle de Brachiaria plantaginea (link) Hitchc , Digitaria horizontalis Willd, Amaranthus hybridus L., Commelina benghalensis L. e Eleusine indica (L.) Gaertn, na cultura do cafeeiro catucaí. Os tratamentos foram constituídos por duas doses $(2500+960$ e 3000+960 g.ha $\left.{ }^{-1}\right)$ da mistura pronta de ametryne+simazine com glyphosate, duas doses $(2500+300$ e $3000+300$ g.ha $\left.{ }^{-1}\right)$ de ametryne+simazine com paraquat comparadas com uma dose $(2500+300$ g.ha ${ }^{-1}$ ) de diuron com paraquat e duas testemunhas sem herbicidas (com e sem capina). Para aplicação dos produtos utilizou-se um pulverizador manual, costal, pressurizado por $\mathrm{CO}_{2}$ a 39 libras/pol ${ }^{2}$, munido de quatro bicos de jato plano, tipo leque, $110.02 \mathrm{XR}$, calibrado para distribuir o correspondente a 200 litros de calda por hectare. O delineamento foi em blocos casualizados, com quatro repetições. As avaliações de controle de plantas daninhas, foram realizadas aos 15, 30, 45 e 60 dias após a aplicação. Concluiu-se que: 1) a mistura de ametryne + simazine com glyphosate, nas doses $2500+960$ g.ha ${ }^{-1}$, controlam com eficácia B. plantaginea, D. horizontalis, A. hybridus e C. benghalensis, até aos 60 DAA e, E. indica até aos 45 DAA; 2) a mistura de ametryne + simazine com glyphosate nas doses 3000+960 g.ha ${ }^{-1}$ foram eficazes no controle de D. horizontalis e C. benghalensis até aos 60 DAA e aos 45 DAA de E. indica e A. hybridus; 3) a mistura de ametryne + simazine com paraquat nas duas doses estudadas $\left(2500+300\right.$ e $3000+300$ g.ha $\left.{ }^{-1}\right)$, controlaram bem $A$. hybridus e $C$. benghalensis até aos 45 DAA; 4) a mistura diuron mais paraquat, foi eficaz no controle de A. hybridus e C. Benghalensis.
\end{abstract}

Palavras-chave: Herbicidas, pós-emergência, diuron, café, coffea arabica

\section{EFFECTS OF A COMMERCIAL MIXTURE OF AMETRYNE PLUS SIMAZINE WITH GLYPHOSATE AND PARAQUAT FOR WEED CONTROL IN COFFEE CROP ABSTRACT}

This research was conducted from 01/06 to 03/08/98 at the location of Irai de Minas, with the objective of evaluating the biological activity of a commercial mixture of ametryne plus simazine with glyphosate and paraquat, for the control of Brachiaria plantaginea, Digitaria horizontalis, Amaranthus hybridus, Commelina benghalensis and Eleusine indica in a Catucai coffee crop. Treatments were constituted of two doses $(2500+960$ and 3000+960 g.ha ${ }^{-1}$ ) of the commercial mixture of ametryne plus simazine with glyphosate, two doses $\left(2500+300\right.$ and $3000+300$ g.ha $\left.{ }^{-1}\right)$ of ametryne plus simazine with paraquat compared with a single dose $\left(2500+300\right.$ g.ha $\left.^{-1}\right)$ of diuron with paraquat and two check treatments with no herbicides (hoeing and without hoeing). It was utilized a back-packed manual sprayer 
pressurized with $\mathrm{CO}_{2}$ at $39 \mathrm{lb} / \mathrm{in}^{2}$, with four tee-jet nozzles $110.02 \mathrm{XR}$, calibrated at a flow rate of 200 liters of the solution per hectare. The experimental design used was a randomized complete-block with four replications. The evaluations of weed control were performed at 15, 30, 45 and 60 days after application. It was concluded that: 1) mixtures of ametryne plus simazine with glyphosate at doses of $2500+960$ g.ha ${ }^{-1}$, control with efficacy B. plantaginea; 2) mixtures of ametryne plus simazine with glyphosate at doses of $3000+960$ were efficient for the control of $D$. horizontalis and $C$. benghalensis up to 60 DAA and up to 45 DAA in the control of E. indica and A. hybridus; 3 ) the mixture of ametryne + simazine with paraquat at both doses studied controls efficiently $C$. benghalensis up to 45 days DAA; 4) the mixture diuron plus paraquat, was efficient for the control of $A$. hybridus and $C$. benghalensis.

Key words: herbicides, postemergence, diuron, coffee, coffea arabica.

\section{INTRODUÇÃO}

O manejo adequado de plantas daninhas na cultura do cafeeiro é muito importante, uma vez que estas interferem tanto na produção, como na qualidade do café, além de serem hospedeiras de pragas, doenças e nematóides, contribuindo para elevadas porcentagens de grãos defeituosos e mal formados e para o aumento dos custos de produção (Oliveira \& Gelmini, (1980).

$\mathrm{Na}$ cafeicultua, os herbicidas são muito importantes para o controle de plantas daninhas, inibindo a sua emergência e, ou, seu desenvolvimento. A mistura de produtos de ação em pré-emergência e pós-emergência poderá ser uma alternativa viável, visando principalmente reduzir os custos e aumentar a eficácia no controle. Produtos como a mistura de ametryne mais simazine, glyphosate e paraquat, são largamente utilizados, estando entre os mais comercializados para a cafeicultura.

De acordo com Souza \& Melles (1986), mistura de herbicidas no tanque do pulverizador ou em formulações têm sido usadas para aumentar o espectro de controle das plantas daninhas, ou diminuir os custos através do emprego de produtos mais baratos ou mesmo através da redução de doses, devido as reações sinérgicas que podem ocorrer, quando se misturam dois ou mais produtos.

Costa (1998) comprovou a eficácia das misturas de ametryne + simazine SC (4,0 e

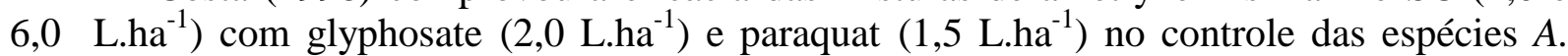
hybridus L., Bidens pilosa L., B. plantaginea, C. benghalensis e E. indica, em aplicação de pós-emergência das plantas daninhas, em jato dirigido, com efeito residual de 62 dias após aplicação dos tratamentos.

Oliveira et al. (1980), estudaram a efetividade da combinação de diversas doses $(0,0$; 0,$6 ; 1,2 ; 2,4 \mathrm{~kg} \cdot \mathrm{ha}^{-1}$ ) do ametryne e simazine, aplicado em pré-emergência no controle de plantas daninhas em lavouras novas de café. Avaliaram 16 tratamentos compostos pela combinação dos referidos herbicidas. Constataram que ambos produtos, quando aplicados individualmente bem como a mistura, proporcionaram bom controle de B. plantaginea, $D$. horizontalis, B. pilosa L e G. parviflora.

Diante do exposto este trabalho teve como objetivo avaliar a eficácia da mistura pronta de ametryne mais simazine com gliphosate e paraquat no controle de plantas daninhas na cultura do cafeeiro.

\section{MATERIAL E MÉTODOS}

O experimento foi conduzido em área comercial de café arábica, variedade 'Catucai', com quatro anos de idade e espaçamento de 4 x $1 \mathrm{~m}$, na Fazenda Cocais, localizada no município de Irai de Minas, MG com $800 \mathrm{~m}$ de altitude, $19^{\circ} 00^{\prime \prime} \mathrm{S}$ de latitude e $47^{\circ} 00^{\prime \prime} \mathrm{W}$ de longitude. 
O solo da área experimental é caracterizado como Latossolo Vermelho-Amarelo, textura argilosa, $32 \%$ de argila e $2,8 \%$ de matéria orgânica. As condições climáticas, durante a condução do trabalho, foram favoráveis para o desenvolvimento da cultura e das plantas daninhas.

O delineamento experimental utilizado foi o de blocos casualizados, com quatro repetições e sete tratamentos conforme descritos na Tabela 1. Cada parcela foi constituída pela área entre duas fileiras de plantas de café, de dois metros de largura e sete metros de comprimento, sendo considerada como bordadura $0,5 \mathrm{~m}$ em cada extremidade.

As aplicações dos herbicidas foram efetuadas quando as plantas daninhas encontravam-se no estádio vegetativo e apresetavam uma altura de 20 a $30 \mathrm{~cm}$ e $90 \%$ da área da parcela coberta ( $40 \%$ de B. plantaginea, $15 \%$ de A. hybridus, $10 \%$ de D. horizontalis, $10 \%$ C. benghalensis, $9 \%$ de E. indica, e $16 \%$ de outras espécies, conforme levantamento prévio). $\mathrm{O}$ equipamento utilizado foi um pulverizador manual, costal, pressurizado por $\mathrm{CO}_{2}$ a 39 libras/pol ${ }^{2}$, munido de quatro bicos de jato plano, tipo leque, $110.02 \mathrm{XR}$, calibrado para distribuir o correspondente a 200 litros de calda por hectare. As condições do ambiente durante a aplicação foram: temperatura de $28^{\circ} \mathrm{C}$, umidade relativa de $69 \%$ e velocidade do vento inferior a $3 \mathrm{~km} / \mathrm{h}$.

As avaliações de controle de plantas daninhas foram realizadas aos 15, 30, 45 e 60 Dias Após a Aplicação (DAA), segundo escala conceitual, em que zero significa nenhum controle e 100 controle total da espécie. Foram feitas avaliações de intoxicação observandose as injúrias causadas às folhas do cafeeiro, localizadas na parte inferior da planta. estatística.

Os dados de porcentagem foram transformados em arco seno $(\%)^{1 / 2}$, para análise

\section{RESULTADOS E DISCUSSÃO}

Os resultados médios de controle de $B$. plantaginea, E indica e D. horizontalis são apresentados nas Tabelas 2,3 e 4 . Verificou-se que apenas a mistura de ametryne mais simazine com glyphosate, nas duas doses estudadas foram eficazes para todas as espécies daninhas avaliadas, apresentando de bom a muito bom controle até aos 45 DAA. Aos 60 DAA, o controle de E. indica foi regular, mas considerado satisfatório, levando em consideração o tempo em que foi realizado (60 dias) e a época do ano (verão), que favorecia a reinfestação da espécie. As demais misturas, com a presença de paraquat, não foram eficazes, propiciando controle abaixo do satisfatórios para as espécies (Tabela 3). O melhor controle apresentado pelas misturas com glyphosate pode ser explicado pelo fato deste herbicida ser um produto sistêmico, que absorvido via foliar é translocado até as raízes, promovendo a morte total das plantas daninhas e com isso não permitindo rebrotar.

Ainda nas Tabelas 2, 3 e 4, observa-se que o controle das plantas daninhas com a menor dose da mistura $\left(2500+960\right.$ g.ha $\left.^{-1}\right)$ não diferiu significativamente da maior dose $\left(3000+960\right.$ g.ha $\left.{ }^{-1}\right)$ nas quatro épocas avaliadas. Estes resultados são importantes, uma vez que, de acordo com Souza \& Melles (1986) as misturas de tanque, pode diminuir os custos, através da redução de doses, devido as reações sinergéticas que podem ocorrer quando se misturam dois ou mais produtos.

Para A. hybridus, todas as misturas dos herbicidas estudados foram eficazes até 45 DAA, ocorrendo uma variação de bom (diuron+paraquat) a excepcional (ametryne+ simazine+glyphosate), enquanto que aos 60 DAA, apenas a mistura com glyphosate na menor dose foi eficaz (Tabela 5).

Com relação a $C$. benghalensis, verificou-se que as misturas com glyphosate a controlaram com eficácia até 60 DAA. Já a misturas de ametryne + simazine com paraquat mostrou resultados irregulares, com controle satisfatório dos 30 aos 45 DAA, ao passo que a de diuron mais paraquat apresentou comportamento menos eficaz e inconsistente (Tabela 6). 
Observando-se as Tabelas 5 e 6, nota-se que as misturas de ametryne + simazine com paraquat e diuron com paraquat foram eficazes somente para A. hybridus e C. benghalensis e até os 45 DAA. Costa (1998), trabalhando com misturas encontraram bons resultados no controle destas espécies até os 62 DAA, quando utilizou a mistura de ametryne mais simazine com paraquat. Em recente trabalho realizado por Bortoletto (2000), no município de Uberlândia, foram obtidos excelentes resultados no controle de $C$. benghalensis com a mistura de diuron + paraquat, em duas aplicações seqüenciais de $500+250 \mathrm{~g}^{\text {. ha }}{ }^{-1}$, até os 67 DAA.

Avaliando a atividade residual da mistura de ametrine + simazine a $2500 \mathrm{~g}^{-h^{-1}} \mathrm{com}$ glyphosate, verificou-se que esta foi muito boa até os 65 DAA para $D$. horizontalis, $B$. plantaginea, A. hybridus e $C$. benghalensis. Resultados semelhantes para as três últimas espécies citadas, foram encontradas por Costa (1998), quando fez uso da mistura de ametryne + simazine SC $\left(4,0\right.$ e 6,0 L.ha $\left.{ }^{-1}\right)$ com glyphosate $\left(2,0\right.$ L.ha $\left.{ }^{-1}\right)$. Já a maior dose da mistura ametryne + simazine, 3000 g.ha ${ }^{-1}+$ glyphosate, foi eficaz até os 65 DAA, apenas para $D$. horizontalis e $C$. benghalensis (Tabelas 2, 4, 5 e 6).

Quanto a sintomas de intoxicação da cultura do cafeeiro, apesar da aplicação ter sido dirigida, foi observada queima das folhas em proporções variáveis, em todos os tratamentos, sem contudo comprometer o desenvolvimento das plantas, no período avaliado.

A mistura de ametryne + simazine com glyphosate, nas doses $2500+960$ g.ha $^{-1}$, controlam com eficácia $B$. plantaginea, D. horizontalis, A. hybridus e C. benghalensi, até aos 60 DAA e $E$. indica até aos 45 DAA. A mistura de ametryne + simazine com glyphosate, nas doses $3000+960$ g.ha ${ }^{-1}$ foram eficazes no controle de $D$. horizontalis e $C$. benghalensis até os 60 DAA e aos 45 DAA para E. indica e A. hybridus. A mistura de ametryne + simazine com paraquat, nas duas doses estudadas $\left(2500+300\right.$ e $\left.3000+300 \mathrm{~g}^{-h^{-1}}\right)$, controlaram bem $A$. hybridus e $C$. benghalensis até aos 45 DAA.A mistura diuron mais paraquat, foi eficaz no controle de A. hybridus e C. benghalensis.

\section{LITERATURA CITADA}

BORTOLLETTO, M. G. Eficácia dos herbicidas glyphosate e paraquat aplicados isolados e em misturas no controle de plantas daninhas na cultura do cafeeiro. Uberlândia: Universidade Federal de Uberlândia, 2000. 30 p. (Monografia de Graduação).

COSTA, E. A. D. Laudo técnico de seletividade e eficiência agronômica do herbicida Topezê SC em mistura de tanque com os herbicidas Roundup e Gramoxone 200, na cultura do café. Campinas: Instituto Biológico, Laboratório da Ciência das Plantas Daninhas, 1998. 23 p.

GUIA DE PRODUTOS DA MONSANTO. São Paulo. Monsanto S.A. 1998. 72p.

OLIVEIRA, E. G.; GELMINI, G. A. Como aplicar herbicidas. Granja, Porto Alegre, v.36, n.390, p. 48-54, 1980.

OLIVEIRA, J. A.; BEGAZO J. C. F. O.; SILVA J. F.; CARDOSO A. A. Combinações de diversas doses de triazinas no controle de plantas daninhas do cafeeiro (Coffea arabica L.). In: CONGRESSO BRASILEIRO DE PESQUISAS CAFEEIRAS, 8, Campos do Jordão, 1980. Resumos... Rio de Janeiro: IBC, 1980. p.403-405.

SOUZA, I. F.; MELlES C. C. A. Controle de plantas daninhas. In: RENA, A.B.; MALAVOLTA, E.; ROCHA, M.; YAMADA T. Cultura do cafeeiro: Fatores que afetam a produtividade. Piracicaba: Potafós, 1986. p 401-408. 
TABELA 1 - Nome comum, nome comercial, doses em gramas do ingrediente ativo e gramas ou litros do produto comercial por hectare. Iraí de Minas, MG. 1998.

\begin{tabular}{llcc}
\hline \multirow{2}{*}{ Nome comum } & \multirow{2}{*}{ Nome comercial } & \multicolumn{2}{c}{ Doses p. c. } \\
\cline { 3 - 4 } & & g.i.a.ha & g ou L.ha $^{-1}$ \\
\hline Ametryne+simazine+glyphosate & Topezê SC+Roundup & $2500+960$ & $5,0+2,0$ \\
Ametryne+ simazine+glyphosate & Topezê SC+Roundup & $3000+960$ & $6,0+2,0$ \\
Ametryne+simazine+paraquat & Topezê SC+Gramoxone & $2500+300$ & $5,0+1,5$ \\
Ametryne+simazine+paraquat & Topezê SC+Gramoxone & $3000+300$ & $6,0+1,5$ \\
Diuron+paraquat & Karmex+Gramoxone & $2500+300$ & $5,0+1,5$ \\
Testemunha capinada & --- & --- & --- \\
Testemunha sem capina & --- & --- & --- \\
\hline
\end{tabular}

TABELA 2 - Controle de Brachiaria plantaginea,, em porcentagem, aos 15, 3045 e 60 dias após a aplicação (DAA) das misturas de herbicidas. Iraí de Minas, MG. 1998.

\begin{tabular}{lcrrrr}
\hline \multirow{2}{*}{ Nome comum } & \multirow{2}{*}{ Dose $\left(\mathrm{g} \cdot \mathrm{ha}^{-1}\right)$} & \multicolumn{4}{c}{ Porcentagem de controle } \\
\cline { 3 - 6 } & & 15DAA & 30DAA & 45DAA & 60DAA \\
\hline Ametryne+simazine+glyphosate & $2500+960$ & $97 \mathrm{ab}$ & $100 \mathrm{a}$ & $100 \mathrm{a}$ & $95 \mathrm{ab}$ \\
Ametryne+ simazine+glyphosate & $3000+960$ & $94 \mathrm{~b}$ & $99 \mathrm{a}$ & $98 \mathrm{a}$ & $81 \mathrm{~b}$ \\
Ametryne+simazine+paraquat & $2500+300$ & $79 \mathrm{c}$ & $86 \mathrm{~b}$ & $49 \mathrm{~b}$ & $37 \mathrm{c}$ \\
Ametryne+simazine+paraquat & $3000+300$ & $71 \mathrm{c}$ & $78 \mathrm{~b}$ & $44 \mathrm{~b}$ & $36 \mathrm{c}$ \\
Diuron+paraquat & $2500+300$ & $35 \mathrm{~d}$ & $66 \mathrm{~b}$ & $31 \mathrm{~b}$ & $30 \mathrm{c}$ \\
Testemunha capinada & --- & $100 \mathrm{a}$ & $100 \mathrm{a}$ & $100 \mathrm{a}$ & $100 \mathrm{a}$ \\
Testemunha sem capina & --- & $0 \mathrm{e}$ & $0 \mathrm{~d}$ & $0 \mathrm{c}$ & $0 \mathrm{~d}$ \\
\hline CV $(\%)$ & & 13,75 & 15,19 & 21,41 & 16,98 \\
\hline
\end{tabular}

Médias seguidas pela mesma letra na coluna, não diferem significativamente pelo teste de Duncan $(\mathrm{P}<$ $0,05)$.

TABELA 3 - Controle de Eleusine indica, em porcentagem, aos 15, 3045 e 60 dias após a aplicação (DAA) das misturas de herbicidas. Iraí de Minas, MG. 1998.

\begin{tabular}{lcrrrr}
\hline \multirow{2}{*}{ Nome comum } & \multirow{2}{*}{ Dose $\left(\mathrm{g} . h a^{-1}\right)$} & \multicolumn{4}{c}{ Porcentagem de Controle } \\
\cline { 3 - 6 } & & 15DAA & $30 \mathrm{DAA}$ & $45 \mathrm{DAA}$ & 60DAA \\
\hline Ametryne+simazine+glyphosate & $2500+960$ & $86 \mathrm{~b}$ & $92 \mathrm{a}$ & $94 \mathrm{~b}$ & $80 \mathrm{~b}$ \\
Ametryne+ simazine+glyphosate & $3000+960$ & $79 \mathrm{~b}$ & $91 \mathrm{a}$ & $94 \mathrm{~b}$ & $79 \mathrm{~b}$ \\
Ametryne+simazine+paraquat & $2500+300$ & $73 \mathrm{~b}$ & $57 \mathrm{~b}$ & $22 \mathrm{c}$ & $07 \mathrm{c}$ \\
Ametryne+simazine+paraquat & $3000+300$ & $69 \mathrm{~b}$ & $56 \mathrm{~b}$ & $18 \mathrm{c}$ & $06 \mathrm{c}$ \\
Diuron+paraquat & $2500+300$ & $47 \mathrm{c}$ & $18 \mathrm{c}$ & $12 \mathrm{c}$ & $06 \mathrm{c}$ \\
Testemunha capinada & --- & $100 \mathrm{a}$ & $100 \mathrm{a}$ & $100 \mathrm{a}$ & $100 \mathrm{a}$ \\
Testemunha sem capina & --- & $0 \mathrm{e}$ & $0 \mathrm{~d}$ & $0 \mathrm{c}$ & $0 \mathrm{~d}$ \\
\hline CV $(\%)$ & & 15,54 & 26,78 & 17,74 & 26,66 \\
\hline
\end{tabular}

Médias seguidas pela mesma letra na coluna, não diferem significativamente pelo teste de Duncan $(\mathrm{P}<$ $0,05)$. 
TABELA 4 - Controle de Digitaria horizontalis, em porcentagem, aos 15, 3045 e 60 dias após a aplicação (DAA) das misturas de herbicidas. Iraí de Minas, MG. 1998.

\begin{tabular}{lccccc}
\hline \multirow{2}{*}{ Nome comum } & \multirow{2}{*}{ Dose $\left(\mathrm{g}^{*} \mathrm{ha}^{-1}\right)$} & \multicolumn{4}{c}{ Porcentagem de controle } \\
\cline { 3 - 6 } & & 15DAA & 30DAA & 45DAA & 60DAA \\
\hline Ametryne+simazine+glyphosate & $2500+960$ & $90 \mathrm{~b}$ & $94 \mathrm{~b}$ & $100 \mathrm{a}$ & $95 \mathrm{ab}$ \\
Ametryne+ simazine+glyphosate & $3000+960$ & $89 \mathrm{~b}$ & $93 \mathrm{~b}$ & $98 \mathrm{a}$ & $91 \mathrm{abc}$ \\
Ametryne+simazine+paraquat & $2500+300$ & $77 \mathrm{c}$ & $83 \mathrm{bc}$ & $53 \mathrm{~b}$ & $67 \mathrm{bc}$ \\
Ametryne+simazine+paraquat & $3000+300$ & $71 \mathrm{c}$ & $81 \mathrm{bc}$ & $47 \mathrm{~b}$ & $52 \mathrm{c}$ \\
Diuron+paraquat & $2500+300$ & $57 \mathrm{~d}$ & $70 \mathrm{c}$ & $25 \mathrm{c}$ & $52 \mathrm{c}$ \\
Testemunha capinada & --- & $100 \mathrm{a}$ & $100 \mathrm{a}$ & $100 \mathrm{a}$ & $100 \mathrm{a}$ \\
Testemunha sem capina & --- & $0 \mathrm{e}$ & $0 \mathrm{~d}$ & $0 \mathrm{~d}$ & $0 \mathrm{~d}$ \\
\hline $\mathrm{CV}(\%)$ & & 8,21 & 13,06 & 14,75 & 28,21 \\
\hline
\end{tabular}

Médias seguidas pela mesma letra na coluna, não diferem significativamente pelo teste de Duncan (P < $0,05)$.

TABELA 5 - Controle de Amaranthus hybridus, em porcentagem, aos 15, 3045 e 60 dias após a aplicação (DAA) das misturas de herbicidas. Iraí de Minas, MG. 1998.

\begin{tabular}{lccccc}
\hline \multirow{2}{*}{ Nome comum } & \multirow{2}{*}{ Dose $\left(\mathrm{g}^{*} \mathrm{ha}^{-1}\right)$} & \multicolumn{4}{c}{ Porcentagem de controle } \\
\cline { 3 - 6 } & & 15DAA & 30DAA & 45DAA & 60DAA \\
\hline Ametryne+simazine+glyphosate & $2500+960$ & $97 \mathrm{~b}$ & $98 \mathrm{~b}$ & $99 \mathrm{ab}$ & $89 \mathrm{~b}$ \\
Ametryne+ simazine+glyphosate & $3000+960$ & $95 \mathrm{~b}$ & $98 \mathrm{bc}$ & $98 \mathrm{abc}$ & $78 \mathrm{bc}$ \\
Ametryne+simazine+paraquat & $2500+300$ & $94 \mathrm{~b}$ & $96 \mathrm{bc}$ & $95 \mathrm{bcd}$ & $71 \mathrm{bc}$ \\
Ametryne+simazine+paraquat & $3000+300$ & $94 \mathrm{~b}$ & $95 \mathrm{bc}$ & $91 \mathrm{~cd}$ & $61 \mathrm{c}$ \\
Diuron+paraquat & $2500+300$ & $93 \mathrm{~b}$ & $93 \mathrm{c}$ & $88 \mathrm{~d}$ & $56 \mathrm{c}$ \\
Testemunha capinada & --- & $100 \mathrm{a}$ & $100 \mathrm{a}$ & $100 \mathrm{a}$ & $100 \mathrm{a}$ \\
Testemunha sem capina & --- & $0 \mathrm{c}$ & $0 \mathrm{~d}$ & $0 \mathrm{e}$ & $0 \mathrm{~d}$ \\
\hline $\mathrm{CV}(\%)$ & & 8,61 & 6,32 & 8,48 & 15,66 \\
\hline
\end{tabular}

Médias seguidas pela mesma letra na coluna, não diferem significativamente pelo teste de Duncan $(\mathrm{P}<$ $0,05)$.

TABELA 6 - Controle de Commelina benghalensis, em porcentagem, aos 15, 3045 e 60 dias após a aplicação (DAA) das misturas de herbicidas. Iraí de Minas, MG. 1998.

\begin{tabular}{lcrrrr}
\hline \multirow{2}{*}{ Nome comum } & \multirow{2}{*}{ Dose $\left({\left.\mathrm{g} . h \mathrm{~h}^{-1}\right)}^{-}\right.$} & \multicolumn{4}{c}{ Porcentagem de Controle } \\
\cline { 3 - 6 } & & 15DAA & 30DAA & 45DAA & 60DAA \\
\hline Ametryne+simazine+glyphosate & $2500+960$ & $90 \mathrm{~b}$ & $90 \mathrm{~b}$ & $94 \mathrm{~b}$ & $91 \mathrm{ab}$ \\
Ametryne+ simazine+glyphosate & $3000+960$ & $85 \mathrm{bc}$ & $89 \mathrm{~b}$ & $92 \mathrm{bc}$ & $85 \mathrm{ab}$ \\
Ametryne+simazine+paraquat & $2500+300$ & $83 \mathrm{bc}$ & $88 \mathrm{~b}$ & $88 \mathrm{~cd}$ & $79 \mathrm{~b}$ \\
Ametryne+simazine+paraquat & $3000+300$ & $83 \mathrm{bc}$ & $78 \mathrm{c}$ & $88 \mathrm{~cd}$ & $68 \mathrm{~b}$ \\
Diuron+paraquat & $2500+300$ & $79 \mathrm{c}$ & $71 \mathrm{c}$ & $86 \mathrm{~d}$ & $53 \mathrm{~b}$ \\
Testemunha capinada & --- & $100 \mathrm{a}$ & $100 \mathrm{a}$ & $100 \mathrm{a}$ & $100 \mathrm{a}$ \\
Testemunha sem capina & --- & $0 \mathrm{~d}$ & $0 \mathrm{~d}$ & $0 \mathrm{e}$ & $0 \mathrm{~d}$ \\
\hline $\mathrm{CV}(\%)$ & & 8,06 & 6,69 & 4,71 & 24,10 \\
\hline
\end{tabular}

Médias seguidas pela mesma letra na coluna, não diferem significativamente pelo teste de Duncan (P < $0,05)$. 